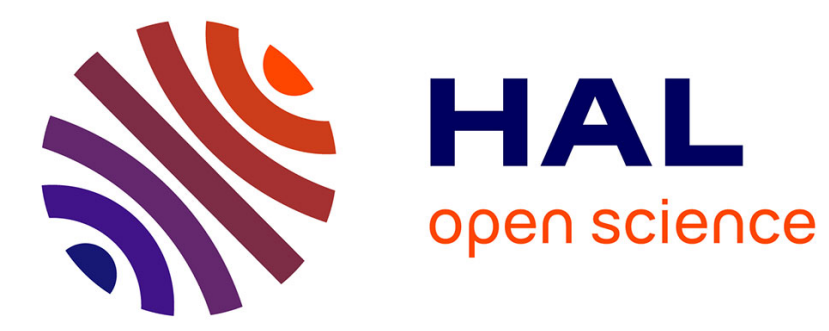

\title{
Wavelet-Galerkin method for periodic heterogeneous media
}

\author{
Serge Dumont, Frédéric Lebon
}

\section{To cite this version:}

Serge Dumont, Frédéric Lebon. Wavelet-Galerkin method for periodic heterogeneous media. Computers \& Structures, 1996, 61 (1), pp.55-65. 10.1016/0045-7949(95)00392-4 . hal-01113791

\section{HAL Id: hal-01113791 \\ https://hal.science/hal-01113791}

Submitted on 31 May 2018

HAL is a multi-disciplinary open access archive for the deposit and dissemination of scientific research documents, whether they are published or not. The documents may come from teaching and research institutions in France or abroad, or from public or private research centers.
L'archive ouverte pluridisciplinaire HAL, est destinée au dépôt et à la diffusion de documents scientifiques de niveau recherche, publiés ou non, émanant des établissements d'enseignement et de recherche français ou étrangers, des laboratoires publics ou privés.

\section{다)(1) $(5$}

Distributed under a Creative Commons Attribution - NonCommercial| 4.0 International 


\title{
WAVELET-GALERKIN METHOD FOR PERIODIC HETEROGENEOUS MEDIA
}

\author{
S. Dumont and F. Lebon \\ Laboratoire de Mécanique et Génie Civil, Université Montpellier 2, P1. E. bataillon, \\ 34095 Montpellier Cedex 5, France
}

\begin{abstract}
This paper is devoted to a new algorithm to compute the local and overall response of a composite. The local elastic problem is discretized by a Galerkin-wavelet method. Details are given to compute the terms of the matrix and of the right hand side. The local solution is selected by a viscosity method. The solution with average equal to zero is chosen. Due to the form of the wavelet bases, the macroscopic coefficients are computed directly from the wavelet coefficients of the solution. Numerical examples are presented. Comparisons are made with analytic solutions and with finite element computations.
\end{abstract}

\section{INTRODUCTION}

Many structural analysis problems are concerned with heterogeneous media. Different approaches have been explored by many authors [1-5] to determine the elastic behaviour of composite materials. A large class of material could be considered as periodic. Theoretical and numerical results have been given in several previous papers [5-11]. The idea is to consider the material being a juxtaposition of identical cells, with the same geometrical and mechanical properties. In elastostatics, it is possible to obtain the macroscopic properties by the knowledge of these microstructures. From a numerical point of view, the classical method to solve this kind of problem, is the finite element method $[7,18]$. Another approach has been proposed using Fourier transform $[8,18,20]$. The difficulty is to eliminate Gibbs phenomena near the heterogeneities; these problematic phenomena could vanish using Fejer series.

In this paper, an original wavelet-Galerkin method is proposed to solve the problem on the microscopic structure. The advantages of the wavelet transform are, in particular, localization and adaptivity [13]. In this case, using a viscosity method [12], it is possible to obtain the displacement solution with the average equal to zero. The coefficients of the matrix are computed using a fast original algorithm based on the product of three wavelets [19]. The evaluation of the right hand side is obtained by the generalization of a method given by Beylkin $[14,15]$.

The paper is organized as follows: in Section 2, we introduce some aspects of the periodic homogenization and we give precise definitions of macroscopic and microscopic variables: we then provide in Section 3 an introduction to viscosity methods, we justify their use for our problem and we review the necessary mathematical background; Section 4 is devoted to the discretization of the problem using wavelet-Galerkin method and to the matrix and the right-hand side coefficients' computations; Section 5 contains some numerical examples: validity is shown comparing results with analytical solutions and with finite element solutions given in literature $[7,18]$.

\section{PERIODIC HOMOGENIZATION SUMMARY}

\subsection{Microscopic and macroscopic variables, notations}

A two phase isotropic elastic composite is considered. We intend to study the behaviour of this heterogeneous media. In this paper, the notion of "equivalent" material is used. By equivalent, we mean that under the same loadings, this equivalent material has globally the same response. In a celebrated paper, Hashin and Strickman [2] have computed optimal bounds for the bulk and shear moduli of a two phase composite. Without going into further detail, these bounds depend on the shear and bulk moduli of the two phases and on the volumic fraction of the two phases in the composite. One could loosely categorize our work in the same framework.

The theory of periodic homogenization focuses on an idealized composite consisting of the juxtaposition of identical heterogeneities. To begin with, then, we give some precisions about notations.

Let $\Omega$ be a given bi-dimensional domain (with Cartesian coordinates $x_{1}, x_{2}$ ) containing the different materials with a periodic representation (see Fig. 1). Let $Y$ be a (periodic) representative part of $\Omega$. We 
distinguish two scales: the macroscopic scale $(x \in \Omega)$ and the microscopic one $(y \in Y)$.

The displacement $\mathbf{U}$ is decomposed into two parts:

$$
\mathbf{U}(\mathbf{x}, \mathbf{y})=E \mathbf{x}+\mathbf{u}(\mathbf{y})
$$

and the deformation tensor $\mathbf{e}$ is written:

$$
\mathbf{e}(\mathbf{U}(\mathbf{x}, \mathbf{y}))=E+\mathbf{e}(\mathbf{u}(\mathbf{y}))
$$

where $E$ is constant and given in $Y$.

We denote (using Einstein's notations):

$$
\mathbf{e}_{k /}(\mathbf{u})=\frac{1}{2}\left(u_{k . l}+u_{i k}\right)
$$

or in other terms

$$
\mathbf{e}(\mathbf{u})=D \mathbf{u}=\operatorname{grad}_{\mathrm{s}} \mathbf{u}
$$

with:

$$
u_{k, t}=\frac{\partial u_{k}}{\partial x_{1}}
$$

$E x$ is the part of the displacement slowly oscillant and $\mathbf{u}(\mathbf{y})$ is the (periodic) part quickly oscillant. $E \mathbf{x}$ represents the displacement in $Y$ if the material is homogeneous. $\mathbf{e}(\mathbf{u})$ represents a fluctuation of the local deformation, then:

$$
\langle\mathbf{e}(\mathbf{U})\rangle_{Y}=E
$$

$\langle\cdot\rangle_{Y}$ is the average on $Y$ :

$$
\langle\mathbf{f}\rangle_{Y}=\frac{1}{\operatorname{mes}(Y)} \int_{Y} f(\mathbf{y}) \mathrm{d} y \text { with } \operatorname{mes}(Y)=\int_{Y} \mathrm{~d} y \text {. }
$$

Thereby:

$$
\langle\mathbf{e}(\mathbf{u})\rangle_{Y}=0
$$

We denote $\Sigma$ the macroscopic stress tensor and $\sigma$ the stress tensor in $Y$.

The macroscopic stress tensor is given by:

$$
\Sigma=\langle\sigma\rangle_{Y}
$$

The material being elastic, we have:

$$
\sigma_{i j}=C_{i j k}\left(\mathbf{e}_{k \prime}(\mathbf{u})+E_{k \prime}\right)
$$

or

$$
\sigma=C(D \mathbf{u}+E)
$$

where $C=C(y)$ is the elasticity tensor on $Y$. We denote $A$, the localization tensor such that:

$$
\mathbf{e}_{i j}(\mathbf{U})=A_{i \not k l} E_{k l}
$$

or

$$
\mathbf{e}(\mathbf{U})=A E
$$

Under these hypotheses, we infer that:

$$
\Sigma=\langle C A\rangle_{\gamma} E
$$

We give in the next sections of this paper a new approach to compute the macroscopic stress tensor.

\subsection{Equilibrium equation in the representative elementary volume}

A fundamental question in heterogeneous media mechanics concerns what happens when a deformation is applied. Merely, the determination of $\Sigma$ corresponding to a given deformation $E$ needs the resolution of the equilibrium equations (EQ) on $Y$

Problem EQ. $E \in\left(L^{2}(Y)\right)^{3}$ be given, find $\mathbf{u} \in\left(\mathrm{H}_{\mathrm{P}}^{1}(\mathrm{Y})\right)^{2}$ such that

$$
\operatorname{div} C D \mathbf{U}=0
$$

or

$$
\operatorname{div} C D \mathbf{u}=-\operatorname{div} C E
$$

or

$$
D^{\mathrm{T}} C D \mathbf{u}=-D^{\mathrm{T}} C E
$$

with $L_{\mathrm{p}}^{2}(Y)=\left\{\mathbf{v} \in L_{\mathrm{loc}}^{2}(Y), \quad \mathbf{v}\left(x_{1}+k_{1}, x_{2}+k_{2}\right)=\right.$ v $\left(x_{1}, x_{2}\right)$ a.e., $\left.k_{1}, k_{2} \in \mathbb{Z}\right\}$

and

$$
H_{\mathrm{p}}^{\prime}(Y)=\left\{\mathbf{v} \in L_{\mathrm{p}}^{2}(Y), \frac{\partial \mathbf{v}}{\partial x_{1}} \in L^{2}(Y), \frac{\partial \mathbf{v}}{\partial x_{2}} \in(Y)\right\} .
$$

$H^{\prime}(Y)$ is the Sobolev space of order one. Details about the periodic conditions on the boundary of the domain $Y$ are given in Section 4.

The macroscopic stress tensor $\Sigma$ is determined by conditions (9), (10), (11) and (14).

It is clear that the solution $\mathbf{u}$ of problem EQ is defined to within a rigid field. In the next section, a mathematical method is introduced to select one, and only one, solution. In particular, it is easy to select a solution with the average equal to zero. Using the fast wavelet transform, it is easier to compute this kind of solution than to impose a given displacement on the boundary of the domain. 


\section{THE VISCOSITY METHOD}

\subsection{Asymptotic results}

In this section, we focus on a theorem due to Attouch [12]. The aim of this theorem consists of replacing a minimization problem, the solution of which is not unique, by approached problems, called viscous problems, where the solutions are unique and converge toward a particular solution of the initial problem: commonly called the selection principle.

Theorem 1. Let $f: X \rightarrow \mathbb{R} \cup\{+\infty\}$ be an extended real-valued function and

$$
\text { (P) } \quad \min \{f(x): x \in X\}
$$

the associated minimization problem.

Let us assume that $\inf _{x} f$ is a finite real number.

Let $g: X \rightarrow \mathbb{R}^{+}$be a nonnegative real-valued function and, for any $\epsilon>0$, let us consider the approximate minimization problem

$$
\left(P_{c}\right) \quad \min \{f(x)+\epsilon g(x): x \in X\}
$$

Let us assume that there exists a topology on $X$ such that the following topological properties (i) and (ii) are satisfied:

(i) for every $\epsilon>0$, there exists a solution $u_{c}$ to $\left(P_{c}\right)$ and the sequence $\min \left\{u_{c}, \epsilon \rightarrow 0\right\}$ is relatively compact;

(ii) $f$ and $g$ are lower semicontinuous.

Then, every limit point $u$ of the sequence $\left\{u_{c}, \epsilon \rightarrow 0\right\}$, minimizes the function $f$ on $X$ and satisfies the viscosity selection principle

$$
\left\{\begin{array}{l}
g(u) \leqslant g(v) \quad \forall v \in \text { argminf } \\
u \in \text { argminf. }
\end{array}\right.
$$

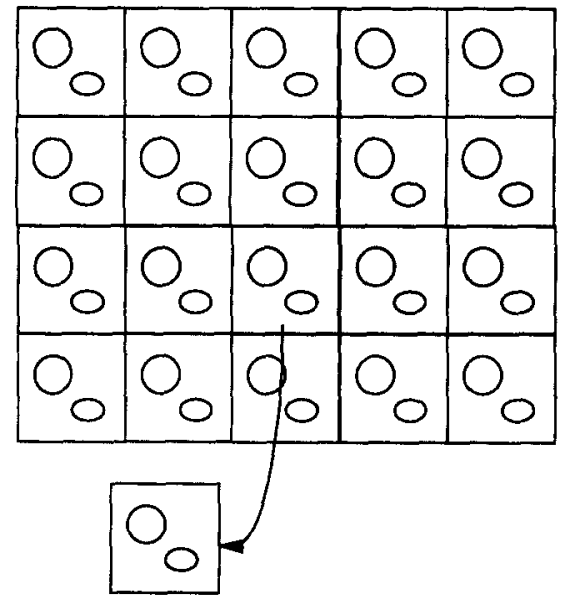

Fig. 1. Periodic heterogeneous media and its representative cell.

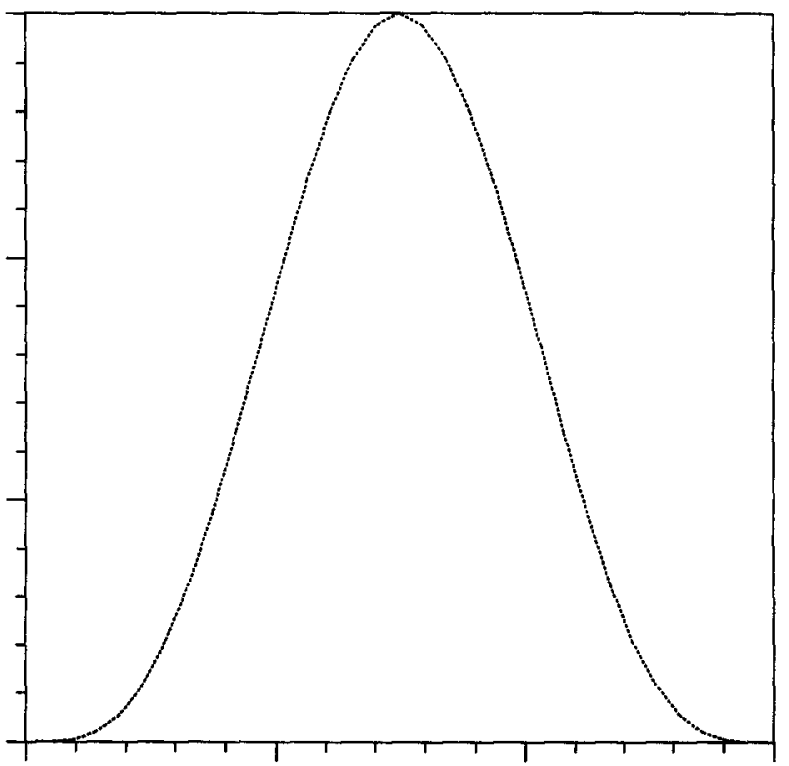

Fig. 2. Comparison between numerical and analytical solutions for $u_{1}\left(0.5, x_{2}\right)$ (validation test). 


\subsection{Application to heterogeneous media}

In this section the former theorem is applied in order to solve the problem on the representative part $Y$ of $\Omega$.

Let us consider the following problem of energy minimization (EM)

$$
\text { (EM) } \quad \min \left\{\frac{1}{2} \int_{Y} \sigma(\mathbf{v}): \mathbf{e}(\mathbf{v}) \mathrm{d} y: \mathbf{v} \in\left(H_{\mathrm{p}}^{\prime}(Y)\right)^{2}\right\} \text {. }
$$

Let us consider the approximate problem (VEM)

$$
\begin{aligned}
\text { (VEM) } \min \{ & \frac{1}{2} \int_{Y} \sigma(\mathbf{v}): \mathbf{e}(\mathbf{v}) \mathrm{d} y \\
& \left.+\frac{\epsilon}{2}\|\mathbf{v}\|_{L^{2}(Y)}^{2}: \mathbf{v} \in\left(H_{\mathrm{p}}^{\prime}(Y)\right)^{2}\right\} .
\end{aligned}
$$

The following corollary is introduced:

Corollary 1. Every limit point $\mathbf{u}$ of the sequence $\left\{\mathbf{u}_{c}, c \rightarrow 0\right\}$, minimizes the function

$$
\frac{1}{2} \int_{Y} \sigma(\mathbf{v}): \mathbf{e}(\mathbf{v}) \mathrm{d} y
$$

on $\left(H_{\mathrm{p}}^{\mathrm{l}}(Y)\right)^{2}$ and satisfies the viscosity selection principle

$$
\left\{\begin{array}{l}
\|\mathbf{u}\|_{L^{2}(\eta)} \leqslant\|\mathbf{v}\|_{L^{2}(\eta} \quad \forall \mathbf{v} \in \operatorname{argmin}\left\{\frac{1}{2} \int_{Y} \sigma(\cdot): \mathbf{e}(\cdot) \mathrm{d} y\right\} \\
\mathbf{u} \in \operatorname{argmin}\left\{\frac{1}{2} \int_{Y} \sigma(\cdot): \mathbf{e}(\cdot) \mathrm{d} y\right\}
\end{array}\right.
$$

\section{Proof}

This result is an elementary application of theorem 1 , with

$$
X=\left(H_{p}^{\mathrm{l}}(Y)\right)^{2}, f(\mathbf{v})=\frac{1}{2} \int_{Y} \sigma(\mathbf{v}): \mathbf{e}(\mathbf{v}) \mathrm{d} y
$$

and

$$
g(\mathbf{v})=\frac{1}{2}\|\mathbf{v}\|_{L^{2}(\eta)}^{2}
$$

An important result gives

Proposition 1. The solution selected by the viscosity selection principle verifies

$$
\langle\mathbf{u}\rangle_{Y}=0
$$

\section{Proof}

The viscosity selection principle gives $\|\mathbf{u}\|_{L^{2}(Y)} \leqslant\|\mathbf{v}\|_{L^{2}(h)} \quad \forall \mathbf{v} \in\left(H_{\mathbf{p}}^{l}(Y)\right)^{2}$.

Then, $\|\mathbf{u}\|_{L^{2} \mid \eta} \leqslant\|\mathbf{u}+\mathbf{C}\|_{L^{2}(\eta)} \quad \forall \mathbf{C} \in \mathbb{R}^{2}$.

In other words,

$$
C^{2}|Y|+2 C \int_{Y} u_{i} \mathrm{~d} y \geqslant 0 \quad \forall C \in \mathbb{R} .
$$

Thus,

$$
\int_{Y} \mathbf{u} d y=0 . \quad \text { Q.E.D. }
$$

The problem (EM) is the elastic energy minimization problem associated to the problem (EQ). These problems are equivalent to the virtual work principle (VW).

Problem $V W$. Find $\mathbf{u} \in\left(H_{\mathrm{p}}^{1}(Y)\right)^{2}$ such that

$$
a(\mathbf{u}, \mathbf{v})=1(\mathbf{v}) \quad \forall \mathbf{v} \in\left(H_{\mathrm{p}}^{1}(Y)\right)^{2}
$$

with

$$
a(\mathbf{u}, \mathbf{v})=\int_{Y} \sigma(\mathbf{u}): \mathbf{e}(\mathbf{v}) \mathrm{d} y=\int_{Y} C D(\mathbf{u}): D(\mathbf{v}) \mathrm{d} y
$$

and

$$
1(v)=-\int_{Y} C E: D(v) \mathrm{d} y .
$$

On the other hand, the viscous energy minimization (VEM) is equivalent to the viscous equilibrium equation (VEQ) and to the viscous virtual work principle (VVW).

Problem VEQ. $E \in\left(L^{2}(Y)\right)^{3}$ given, find $\mathbf{u} \in\left(H_{\mathrm{p}}^{\mathrm{l}}(Y)\right)^{2}$ and $\sigma \in\left(L_{\mathrm{ap}}^{2}(Y)\right)^{9}$ such that

$$
\operatorname{div} \sigma+\epsilon \mathbf{u}=0
$$

with

$$
\sigma=C(D \mathbf{u}+E) .
$$

Problem VVM. Find $\mathbf{u} \in\left(H_{\mathrm{p}}^{\mathrm{L}}(Y)\right)^{2}$ such that

$$
a_{c}(\mathbf{u}, \mathbf{v})=1(\mathbf{v}) \quad \forall \mathbf{v} \in\left(H_{\mathrm{p}}^{\prime}(Y)\right)^{2}
$$

with

$$
a_{c}(\mathbf{u}, \mathbf{v})=\int_{Y} \sigma(\mathbf{u}): \mathbf{e}(\mathbf{v}) \mathrm{d} y+\epsilon \int_{Y} \mathbf{u} \cdot \mathbf{v} \mathrm{d} y .
$$

The solution of problems (VEQ), (VEM) and 
(VVM) converges towards the solution of problem (EQ) such that

$$
\int_{Y} \mathbf{u} \mathrm{d} y=0
$$

To summarize, the process chosen to solve the equilibrium equation (EQ) could be described concisely as the following: the problem (EQ) is replaced by the problem (VEQ) which is discretized using a wavelet-Galerkin method; the solution of this problem selected by the viscosity selection principle converges toward the solution of problem (EQ) with average equal to zero.

In the following section, we present the discretization of the problem by a Galerkin-wavelet method using Daubechies wavelets, which are compactly supported wavelet bases.

\section{THE WAVELET-GALERKIN METHOD}

\subsection{One-dimensional Daubchies wavelets}

In this section, we present a brief summary of one-dimensional Daubechies wavelets [13]. Let $M$ be a positive integer. There exists a sequence of real numbers $\{h(n)\}_{n=0, \ldots 2 M-1}$ such that

$$
\sum_{n=0}^{2 M-1} h(n)=\sqrt{2}
$$

and

$$
\sum_{n=0}^{n=2 M-1} h(n) h(n+2 k)=\delta_{0 k}, \quad \forall k \in \mathbb{Z} .
$$

This sequence defines two compactly supported functions $\varphi$, called the scale function and $\Psi$, called the associated wavelet. These functions verify

$$
\begin{aligned}
& \varphi(x)=\sqrt{2} \sum_{n=0}^{n=2 M-1} h(n) \varphi(2 x-n), \quad \forall x \in \mathbb{R} \\
& \Psi(x)=\sqrt{2} \sum_{n=0}^{n-2 M-1} g(n) \varphi(2 x-n), \quad \forall x \in \mathbb{R}
\end{aligned}
$$

where

$$
g(n)=(-1)^{n} h(2 M-n-1), n=0, \ldots, 2 M-1 .
$$

$$
\int_{\mathbb{R}} \varphi(x) \varphi(x-k) \mathrm{d} x=\delta_{0 k}, \quad \forall k \in \mathbb{Z}
$$

$$
\begin{gathered}
\int_{\mathbb{R}} \Psi(x) \Psi(x-k) \mathrm{d} x=\delta_{0 k}, \quad \forall k \in \mathbb{Z} \\
\int_{\mathbb{R}} \varphi(x) \mathrm{d} x=1 \quad \text { and } \sum_{k \in \mathbb{Z}} \varphi(x-k)=1, \quad \forall x \in \mathbb{R} .
\end{gathered}
$$

The compact support of $\varphi$ and $\Psi$ is included in the closed interval $[0,2 M-1]$.

Let

$$
\varphi_{j k}(x)=2^{j / 2} \varphi\left(2^{j} x-k\right), \quad \forall x \in \mathbb{R}, \forall j, k \in \mathbb{Z},
$$

and

$$
\Psi_{j k}(x)=2^{j / 2} \Psi\left(2^{j} x-k\right), \quad \forall x \in \mathbb{R}, \forall j, k \in \mathbb{Z},
$$

These functions are obtained from $\varphi$ and $\Psi$ using dilatation and translation. The compact support of $\varphi_{j k}$ and $\Psi_{j k}$ is included in the closed interval $\left[k / 2^{j},(k+2 M-1) / 2\right]$.

We define $V$, the closure of the space generated by $\left\{\varphi_{j k}, k \in \mathbb{Z}\right\}$.

The sub-spaces $V$, verify the following conditions:

$$
\begin{gathered}
V_{j} \subset V_{j+1} \\
\bigcap_{i \in \mathbb{Z}} V_{j}=\{0\}
\end{gathered}
$$

and

$$
L^{2}(\mathbb{R})=\bigcup_{j \in \mathbb{Z}} V_{j}
$$

The set of spaces $V_{j}$ is called a multiresolution analysis of $L^{2}(\mathbb{R})$.

\subsection{Periodic wavelets}

Due to the form of the problem, it is necessary to define periodic wavelets and a multiresolution analysis of $L_{\mathrm{p}}^{2}(Y)$.

We define the periodic function $\phi_{j k}$ such that

$$
\phi_{j k}(x)=2^{\prime / 2} \sum_{r \in \mathbb{Z}} \varphi\left(2^{\prime}(x+r)-k\right) .
$$

One can show [17] that the closure of $\left\{\phi_{j k}, k \in \mathbb{Z}\right\}$, noted $V_{j}^{\mathrm{p}}$ defines a multiresolution analysis of $L_{\mathrm{p}}^{2}(0,1)$.

Furthermore, we can determine orthonormal bases of $L_{\mathrm{p}}^{2}(Y)$.

$$
L_{\mathrm{p}}^{2}(Y)=\underset{j}{\mathrm{U}} \mathbb{V}_{j}^{\mathrm{p}}
$$

where $\mathbb{V}_{p}^{p}$ is the closure of the space generated by $\left\{\Phi_{k_{1} k_{2}}^{\prime}, k_{1}, k_{2} \in \mathbb{Z}\right\}$, where

$$
\Phi_{k_{1} k_{2}}^{\prime}\left(x_{1}, x_{2}\right)=\phi_{j k_{1}}\left(x_{1}\right) \phi_{j k_{2}}\left(x_{2}\right) .
$$


It is clear that $\left\{\Phi_{k_{1} k_{2}}^{j}, k_{1}, k_{2} \in \mathbb{Z}\right\}$, is an orthonormal basis of $V_{i}^{p}$.

With these notations, we obtain

$$
\left(L_{\mathfrak{p}}^{2}(Y)\right)^{2}=\left(U \mathbb{V}_{j}^{\mathfrak{p}}\right)^{2} .
$$

If we denote $\left\{\mathbb{V}_{j}, j \in \mathbb{Z}\right\}$ the multiresolution analysis of $\left(L_{\mathrm{p}}^{2}(Y)\right)^{2}$, then an element of $\mathbb{V}_{j}$ is written

$$
\mathbf{u}\left(x_{1}, x_{2}\right)=\left(\begin{array}{c}
u_{1}\left(x_{1}, x_{2}\right) \\
u_{2}\left(x_{1}, x_{2}\right)
\end{array}\right)
$$

with

$$
u_{i}\left(x_{1}, x_{2}\right)=\sum_{\left(k_{1}, k_{2}\right)=0(0,0)}^{\left(k_{1} \cdot k_{2}\right)=\left(N, N^{\prime}\right)} u_{i}^{k_{1}, k_{2}} \Phi_{k_{1}, k_{2}}\left(x_{1}, x_{2}\right)
$$

with $N^{j}=2^{\prime}-1$.

\subsection{Elastostatics discretization}

The problem VVW is discretized using a Galerkin method written in the "wavelet basis" defined in Section 4.2. Then, we obtain the proposition 2 .

Proposition 2. For plane elasticity, the projection of the elasticity operator into the "wavelet basis" with scale equal to $j$, is written. $\mathbf{K}=\mathbf{K}_{(11,12), k 1, k 2) \text {, }}$ $\mathbf{K}$ is a symmetric matrix of order $2^{2 i+1}$, where $\mathbf{K}_{(i 1,12), k 1, k 21}=\mathbf{K}_{i k}$ is an elementary matrix of order 2:

$$
\mathbf{K}_{i k}=\left[\begin{array}{ll}
F_{11}^{11}+F_{22}^{33} & F_{12}^{12}+F_{21}^{33} \\
F_{21}^{12}+F_{12}^{33} & F_{22}^{22}+F_{11}^{33}
\end{array}\right],
$$

where

$$
F_{\mathrm{A}}^{\mathrm{P}}=\int_{Y} C_{\alpha \beta}\left(x_{1}, x_{2}\right) \Phi_{\mathrm{i}, \eta}^{\mathrm{i}}\left(x_{1}, x_{2}\right) \Phi_{\mathbf{k}, \zeta}^{j}\left(x_{1}, x_{2}\right) \mathrm{d} x_{1} \mathrm{~d} x_{2}
$$

with $\mathbf{A}=(\alpha, \beta), \alpha, \beta=1,2$ or 3 and $\mathbf{P}=(\eta, \xi), \eta$, $\xi=1$ or 2 .

For the proof see Ref. [19].

It is clear, from the form of the elementary matrix, that the matrix $\mathbf{K}$ is symmetric. Due to the orthogonality of the wavelet basis, the discretization of the term $\epsilon \int_{Y} \mathbf{u} \cdot \mathbf{v} \mathrm{d} y$ gives us $\epsilon \mathbf{I} d$, where $\mathbf{I} d$ is the identity matrix of order $2^{2 j+1}$. Then the matrix of the problem is equal to $\mathbf{K}+\epsilon \mathbf{I} d$. In the next section, we propose a technique to compute the terms of the matrix $\mathbf{K}$.

4.4. The first case: $C$ is a constant function on the intersection of the supports of $\Phi_{i}^{i}$ and $\Phi_{\mathbf{k}}^{i}$

Equation (51) gives us

$$
F_{\mathrm{A}}^{\mathrm{o}}=C_{\alpha \beta} A_{\mathrm{ik}} B_{\mathrm{ik}},
$$

where

$$
\begin{aligned}
& \text { if } \eta=1 \text { then } n 1=1 \text { and } n 2=0 \\
& \text { if } \eta=2 \text { then } n 1=0 \text { and } n 2=1 \\
& \text { if } \xi=1 \text { then } m 1=1 \text { and } m 2=0 \\
& \text { if } \xi=2 \text { then } m 1=0 \text { and } m 2=1 \\
& A_{\mathbf{i k}}=\int_{0}^{1} \phi_{j i 1}^{(n) 1}\left(x_{1}\right) \phi_{j k 1}^{(m 1)}\left(x_{1}\right) \mathrm{d} x_{1}
\end{aligned}
$$

$$
B_{\mathrm{ik}}=\int_{0}^{1} \phi_{j 2}^{(n 2)}\left(x_{2}\right) \phi_{j k 2}^{(m 2)}\left(x_{2}\right) \mathrm{d} x_{2}
$$

and $f^{(s)}$ is the derivative of order $s(s=0,1,2)$.

Thus, we have to compute the scalar product of two wavelets and their derivatives. The algorithm to compute these terms is given [19] and is based on a theorem given by Beylkin [14]. These terms are computed via filter banks as eigenvectors of a low order matrix.

4.5. The second case: $C$ is not a constant function on the intersection of the supports of $\Phi_{i}^{i}$ and $\Phi_{k}^{i}$

The function $C$ is decomposed into a wavelet basis which is not necessarily the same as in Section 4.2. Due to the form of $C_{x \beta}$, the best choice seems to be the Haar basis (corresponding to $M=1$ ). Let $\theta$ be the wavelet basis of decomposition (order $\mathbb{M}$ ) of $C_{x \beta}$. Then

$$
C_{x \beta}\left(x_{1}, x_{2}\right)=\sum_{\left.\left(\rho_{1}, \rho_{2}\right)=10.0\right)}^{\left(\rho_{1}, \rho_{2}\right)=\left(N^{j}, N^{\prime}\right)} C_{\rho_{1}, \rho_{2}}^{\alpha \beta} \theta_{J \rho_{1}}\left(x_{1}\right) \theta_{J \rho_{2}}\left(x_{2}\right)
$$

with $N^{J}=2 J-1$.

$J$ is not necessarily equal to $j$, but usually $J \geqslant j$ is chosen to have $2^{J-j}$ in $\mathbb{N}$.

Proposition 3 [19]. The evaluation of terms $F_{A}^{\mathrm{P}}$ needs to compute the elementary terms $\Gamma_{J_{t, 4}}^{p}$, where

$$
\Gamma_{J_{j \mathrm{ik}}}^{\mathrm{p}}=\int_{0}^{1} \theta_{J_{k}}(x) \phi_{j i}^{(n)}(x) \phi_{j 0}^{(m)}(x) \mathrm{d} x .
$$

More precisely,

$$
F_{\mathrm{A}}^{\mathrm{P}}=\sum_{\left(\rho_{1}, \rho_{2}\right)=(0,0)}^{\left(N^{\prime}, N^{\prime}\right)} C_{\rho_{1}, \rho_{2}}^{A} \Gamma_{j j r_{1} s_{1}}^{\rho^{1}} \Gamma_{j j_{2} r_{2}}^{\mathrm{p}^{2}}
$$

with $r_{i}=i_{i}-k_{i}$ and $s_{i}=\rho_{i}-2^{j-i} k_{i}$.

Thus, we have to compute elementary terms which can be seen as the "product of three wavelets". The 
algorithm to compute these terms is given [19]. They are given as eigenmatrices of a fourth order tensor.

\subsection{Right hand side computation}

This part of the paper is concerned with performing the right hand side $1(v)$.

Equation (28) gives us $1(\mathbf{v})=-\int_{Y} C E$ : $\mathbf{e}(\mathbf{v}) \mathrm{d} y$.

Let $T=C E$. If

$$
\begin{aligned}
\mathbf{v} & =\left(\begin{array}{c}
\Phi_{i}^{\prime} \\
0
\end{array}\right) \text { then } \int_{Y} C E: \mathbf{e}(\mathbf{v}) \mathrm{d} y \\
& =\int_{Y}\left(T_{11} \Phi_{i, 1}^{\prime}+T_{12} \Phi_{i, 2}^{\prime}\right) \mathrm{d} y
\end{aligned}
$$

and if

$$
\begin{aligned}
\mathbf{v} & =\left(\begin{array}{c}
0 \\
\Phi_{i}^{\prime}
\end{array}\right) \text { then } \int_{Y} C E: \mathbf{e}(\mathbf{v}) \mathrm{d} y \\
& =\int_{Y}\left(T_{12} \Phi_{t, 1}^{\prime}+T_{22} \Phi_{t .2}^{\prime}\right) \mathrm{d} y
\end{aligned}
$$

Then, projection of the right hand side into the wavelet basis is a vector $B$. The elementary vector of order $2, B_{i}=B_{1_{1}, i_{2}}$, is written

$$
B_{i_{1}, i_{2}}=\left(\begin{array}{l}
G_{11}^{10}+G_{12}^{01} \\
G_{12}^{10}+G_{22}^{01}
\end{array}\right)
$$

with

$$
\begin{array}{r}
G_{\mathbf{A}}^{\mathbf{P}}=G_{\mathrm{Ajk}}^{\mathbf{P}}=\int_{Y} T_{x \beta}\left(x_{1}, x_{2}\right) \phi_{j}^{(n)}\left(x_{1}\right) \phi_{j \mathbf{k}}^{(\xi)}\left(x_{2}\right) \mathrm{d} x_{1} \mathrm{~d} x_{2}, \\
\mathbf{P}=(\eta, \xi) \quad \text { and } \quad \mathbf{A}=(\alpha, \beta) .
\end{array}
$$

Due to the choice of $E$ for the computations,

$$
E=\left(\begin{array}{l}
1 \\
0 \\
0
\end{array}\right),\left(\begin{array}{l}
0 \\
1 \\
0
\end{array}\right) \text { or }\left(\begin{array}{l}
0 \\
0 \\
1
\end{array}\right),
$$

we need to compute terms such $F$ in Section 4.3.

As for the computation of $F$, there are two cases. The first case is if $C$ is constant on the intersection of the supports of $\phi_{i i}$ and $\phi_{i k}$. Then, due to the definition of $\phi$, the terms $G$ are equal to zero.

If $C$ is not constant on the intersection of the supports of $\phi_{i j}$ and $\phi_{j k}$, then we introduce as in eqn (56),

$$
T_{x \beta}\left(x_{1}, x_{2}\right)=\sum_{\left(p_{1}, p_{2}\right)=(0.0)}^{\left\langle p_{1}, p_{2}\right)=\left(N^{J}, N^{\prime}\right\}} T_{p_{1}, p_{2}}^{\alpha \beta} \theta_{J p_{1}}\left(x_{1}\right) \theta_{J p_{2}}\left(x_{2}\right)
$$

Then

$$
G_{\mathrm{A}}^{\mathrm{P}}=\sum_{\mathrm{p}=(0,0)}^{(N, N)} T_{\mathrm{p}}^{\alpha \beta} A_{\mathrm{lq}} B_{\mathrm{lp}}
$$

with

$$
\begin{aligned}
& A_{\mathrm{ip}}=\int_{0}^{1} \theta_{J p_{1}}\left(x_{1}\right) \phi_{j i 1}^{(n)}\left(x_{1}\right) \mathrm{d} x_{1} \\
& B_{\mathrm{ip}}=\int_{0}^{1} \theta_{J p_{2}}\left(x_{2}\right) \phi_{j i 2}^{(n)}\left(x_{2}\right) \mathrm{d} x_{2}
\end{aligned}
$$

The coefficients $A$ and $B$ are similar to the terms $A$ and $B$ in Section 4.4, but with two different wavelets. A method to perform these values is given in Section 4.7.

\subsection{Evaluation of two wavelet products in the right} hand side

Using a change of variable, it is clear that

$$
\int_{0}^{1} \theta_{J p}(x) \phi_{j i}^{(n)}(x) \mathrm{d} x=\int_{0}^{1} \theta_{J s}(x) \phi_{j 0}^{(n)}(x) \mathrm{d} x
$$

with $s=\rho-2^{j-} i$.

Then, we have to evaluate the terms

$$
r_{j j k}^{n}=\int_{0}^{1} \theta_{J_{k}}(x) \phi_{j 0}^{(n)}(x) \mathrm{d} x
$$

The two wavelets verify the following conditions:

$$
\theta(x)=\sqrt{2} \sum_{n=0}^{n-2 M-1} H(n) \theta(2 x-n), \quad \forall x \in \mathbb{R}
$$

$$
\varphi(x)=\sqrt{2} \sum_{n=0}^{n=2 M-1} h(n) \phi(2 x-n), \quad \forall x \in \mathbb{R}
$$

Then, the following result is obtained.

\section{Theorem 2.}

$$
r_{00 \alpha}^{n}=2 \sum_{\beta=2}^{\alpha M-2} \alpha=0 \mathbb{D}_{\alpha \beta} r_{00 \beta}^{n}, \quad \alpha=1,2 M-2
$$

with

$$
\mathbb{D}_{2 \beta}=\sum_{n=0}^{x N-1} n=0 H(n) h
$$

$$
\times(\alpha-n-\beta) \chi_{[\beta+n-2 M+1 . \beta+n]}(\alpha) .
$$




\section{Proof}

The proof of this theorem is an elementary development of the eqn (68) using eqns (69) and (70) and a change of variable in $h$. The term $\chi$ appears because $r_{i}=0$ for $i \notin[0,2 M-2]$. Q.E.D.

Tables for $n=1$ are given in Appendix A. Note that

$$
r_{J j k}^{n}=2^{j n} r_{00 k}^{n}
$$

Then, the coefficients for $j<J$ are given using the pyramidal algorithm presented for example in Refs $[17,19,21]$.

\subsection{Evaluation of the macroscopic coefficients}

The aim of this paragraph is to compute, using the wavelet coefficients of the microscopic displacement u, the macroscopic stress tensor $\Sigma$ and the homogenized matrix $\langle C A\rangle_{Y}$.

Due to the definition of $\Sigma$ in eqn (19),

$$
\Sigma=\langle C \mathbf{e}(\mathbf{U})\rangle_{Y}=\langle C \mathbf{e}(\mathbf{u})\rangle_{Y}+\langle C E\rangle_{Y} .
$$

The term $\langle C E\rangle_{Y}$ is a given constant, we need to compute the term $\langle C \mathbf{e}(\mathbf{u})\rangle_{\gamma}$.

Let $\Lambda^{\mu}=\left[0, N^{\mu}\right]^{2}$, for $\mu=J$ or $j$. Equations (48), (49) give us

$$
\begin{aligned}
u_{x}\left(x_{1}, x_{2}\right)= & \sum_{\left(k_{1}, k_{2}\right) \in A^{\prime}} u_{x}^{k_{1}, k_{2}} \\
& \quad \times \phi_{j k_{1}}\left(x_{1}\right) \phi_{j k_{2}}\left(x_{2}\right), \quad \alpha=1,2 .
\end{aligned}
$$

Then, by a simple derivation, we obtain $\mathbf{e}(\mathbf{u})$.

$$
\begin{array}{r}
\mathbf{e}_{i i}(\mathbf{u})=\sum_{\left(k_{1}, k_{2} \mid \in A^{\prime}\right.} u_{i}^{k_{1}, k_{2}} \phi_{j k_{1}}^{(n)}\left(x_{1}\right) \phi_{j k_{2}}^{(m)}\left(x_{2}\right) \\
\quad i=1 \text { or } 2, n=\delta_{i 1} \text { and } m=\delta_{2 i}
\end{array}
$$

and

$$
\begin{aligned}
\mathbf{e}_{12}(\mathbf{u})=\frac{1}{2} \sum_{\left(k_{1}, k_{2}\right) \in A^{i}} u_{i}^{k_{1}, k_{2}} \phi_{j k_{1}}\left(x_{1}\right) \phi_{j k_{2}}^{(!)}\left(x_{2}\right), \\
+\frac{1}{2} \sum_{\left(k_{1}, k_{2}\right) \in A^{j}} u_{2}^{k_{1}, k_{2}} \phi_{j k_{1}}^{(!)}\left(x_{1}\right) \phi_{j k_{2}}\left(x_{2}\right)
\end{aligned}
$$

So, the term $\langle C \mathrm{e}(\mathrm{u})\rangle_{Y}$ is deduced by a decomposition of $C$, if necessary, into the wavelet basis.

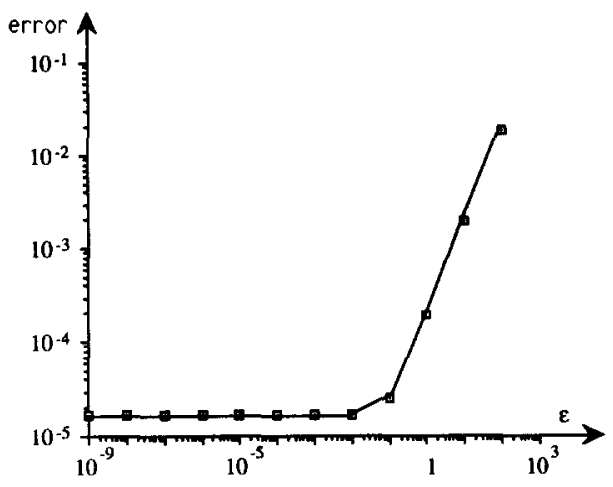

Fig. 3. Fluctuation of the error during the variations of the viscosity (validation test $M=5, j=5$ ).

For example

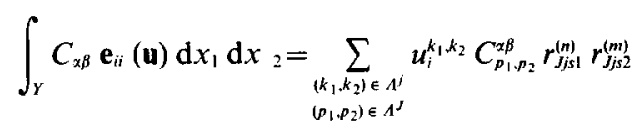

with $s_{\mu}=k_{\mu}-2^{J-1} p_{\mu}$.

Then, it is possible to deduce $\Sigma$ and the symmetric tensor $C^{\mathrm{hom}}=\langle C A\rangle_{\mathrm{Y}}$. For example, if $E=(1,0,0)$ is chosen, then:

$$
\left[\begin{array}{l}
C_{11}^{\text {hom }}=\Sigma_{11}=\left\langle C_{11}\left(e_{11}+1\right)+C_{12} e_{22}\right\rangle_{Y} \\
C_{21}^{\text {hom }}=\Sigma_{22}=\left\langle C_{12}\left(e_{11}+1\right)+C_{22} e_{22}\right\rangle_{Y} \\
C_{31}^{\text {hom }}=\Sigma_{12}=\left\langle C_{33} e_{33}\right\rangle_{Y}
\end{array}\right] .
$$

\section{NUMERICAL APPLICATIONS}

\subsection{Validation tests}

The previous algorithms are validated on an academic homogeneous medium. This test is very useful to give us the parameters to introduce in the method: viscosity coefficient, orders of the wavelets, dimension of the approximation space, numerical method to inverse the linear system.

The right-hand side $f$ is given by

$$
\begin{aligned}
f_{1}(x . y)= & -4 C_{33} x^{4}(1-x)^{4} y^{2}(1-y)^{2} \\
& \times\left(14 y^{2}-14 y+3\right)-4 C_{11} x^{2}(1-x)^{2} y^{4} \\
& \times(1-y)^{4}\left(14 x^{2}-14 x+3\right)-16 \\
& \times\left(C_{12}+C_{33}\right) x^{3}(1-x)^{3} y^{3} \\
& \times(1-y)^{3}(1-2 x)(1-2 y)
\end{aligned}
$$


(a)

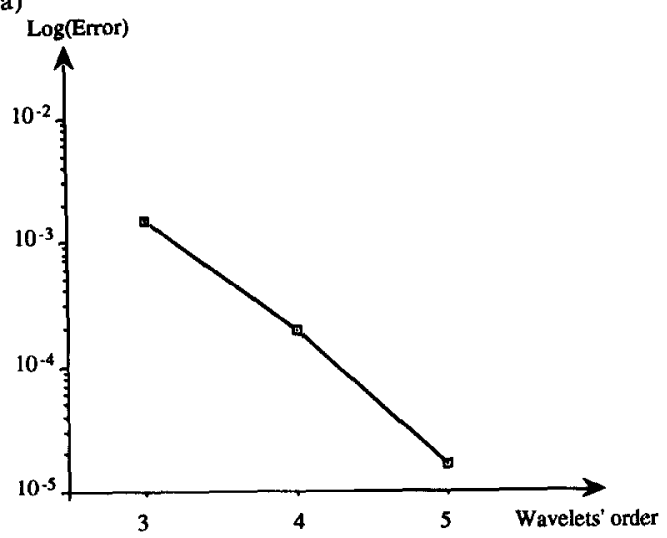

(b)

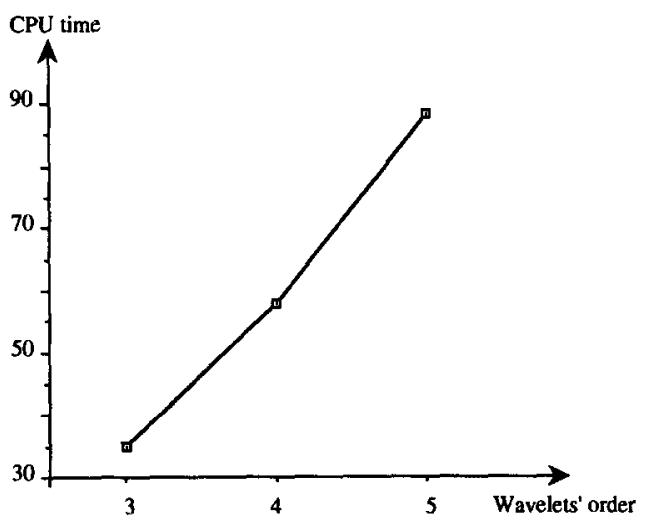

Fig. 4. (a) Error (log). (b) CPU time (Sun Sparc server $630 \mathrm{mp}$ ). Error and CPU time for different order of wavelets (validation test $j=5, f=0.000001$ ).

$$
\begin{aligned}
\mathrm{f}_{2}(x, y)= & -4 C_{33} x^{2}(1-x)^{2} y^{4}(1-y)^{4} \\
& \times\left(14 x^{2}-14 x+3\right)-4 C_{22} x^{4}(1-x)^{4} y^{2} \\
& \times(1-y)^{2}\left(14 y^{2}-14 y+3\right) \\
& -16\left(C_{12}+C_{33}\right) x^{3}(1-x)^{3} y^{3}(1-y)^{3} \\
& \times(1-2 x)(1-2 y)
\end{aligned}
$$

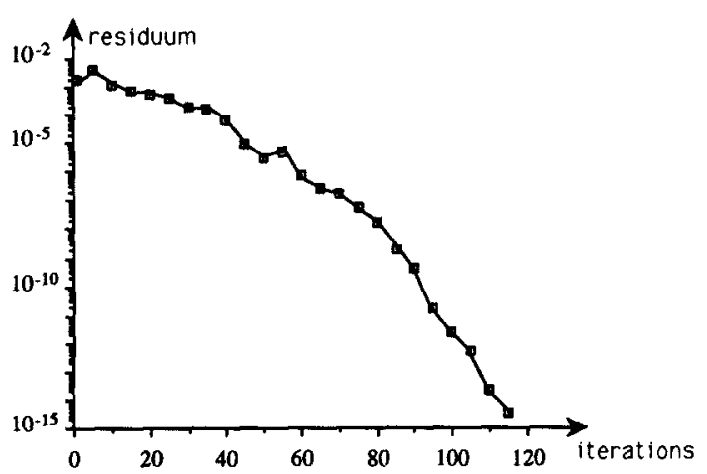

Fig. 5. Evolution of the residuum along the iterative process (validation test $M=5, J=5, \epsilon=0.01$ ).

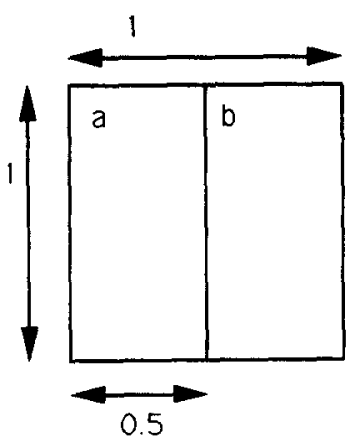

Fig. 6. Two layers composite. (a) Young's modulus: $410 \mathrm{GPa}$, Poisson's ratio: 0.19. (b) Young's modulus: $72 \mathrm{GPa}$, Poisson's ratio: 0.32 .

and the displacement $u$ by

$$
\begin{aligned}
& u_{1}\left(x_{1}, x_{2}\right)=x^{4}(1-x)^{4} y^{4}(1-y)^{4}-\frac{1}{630^{2}} \\
& u_{2}\left(x_{1}, x_{2}\right)=x^{4}(1-x)^{4} y^{4}(1-y)^{4}-\frac{1}{630^{2}}
\end{aligned}
$$

The Young's modulus is equal to $210,000 \mathrm{MPa}$ and the Poisson's ratio to 0.2 (plane stress hypothesis).

Figure 2 shows the accuracy between the numerical results and the analytical solution given by eqns (81), (82). Figure 3 shows the fluctuation of the error during the variations of the viscosity parameter. For this example the value 0.01 is optimal.

Figure 4 shows the solutions for different order of wavelets. It is not necessary to choose a wavelet with too large an order. In this case, the order equal to three gives us a very good approximation of the solution. On the other hand, the level of approximation is chosen equal to 5 , then the number of "nodes" is equal to 1024 . The improvement is not very important for a larger number of nodes.

The linear system is solved by a conjugate gradient method without preconditioner. A sparse matrix storage is used. An important remark concerns the fluctuations of the residuum along the iterative process (see Fig. 5). These fluctuations are very slow and the number of iterations is not too large, so the matrices are not ill-conditioned ones.

\subsection{Two layer composite}

We test the previous approaches on a two layer composite (see Fig. 6) which has the advantage of being elementary with an analytical solution.

For the first layer, the Young's modulus is equal to

Table 1. Macroscopic coefficients (MPa) (two layer composite, plane stress hypothesis, $M=3, j=5$ )

$$
C^{\text {hom }}=\left[\begin{array}{lll}
136000 & 34500 & 0.255 \cdot 10^{-5} \\
34500 & 250000 & 0.276 \cdot 10^{-4} \\
0.725 .10^{-5} & 0.131 \cdot 10^{-4} & 47400
\end{array}\right]
$$


Table 2. Macroscopic coefficients (MPa) (two layer composite, plane stress hypothesis, finite element method)

$C^{\text {hom }}=\left[\begin{array}{lcc}135000 & 34400 & -0.489 \cdot 10^{-10} \\ 34440 & 250000 & -0.734 \cdot 10^{-11} \\ 0.584 \cdot 10^{-10} & -0.824 \cdot 10^{-11} & 47100\end{array}\right]$

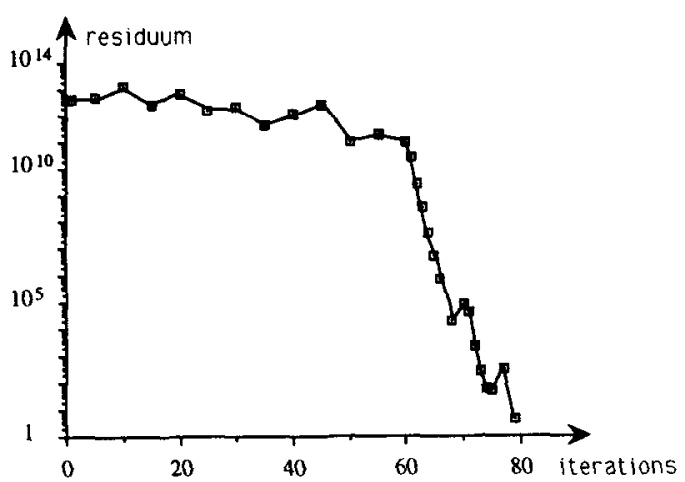

Fig. 7. Evolution of the residuum along the iterative process (two layer composite $M=3, j=5, \epsilon=0.01$, plane strain hypothesis).

$410,000 \mathrm{MPa}$ and the Poisson's ratio to 0.19 (Silicium). For the second layer, the Young's modulus is equal to $72,000 \mathrm{MPa}$ and the Poisson's ratio to 0.32 (Aluminium).

The numerical results are given in Table 1 and give us a good accuracy between the analytical solution (no more than $0.1 \%$ ). Table 2 shows results given by a finite element method.

The optimal value of the viscosity coefficient is equal to 0.01 . The fluctuations of the residuum are given in Fig. 7. Without preconditioner, the number of iterations is not too large and the fluctuations are due to the difference of elasticity coefficients.

\subsection{Fiber-matrix composite}

The second example concerns a fiber-matrix composite (see Fig. 8). The numerical results are compared with a finite element method [18].

The materials are the same as for paragraph 5.2.: silicium for the fiber and aluminium for the matrix.

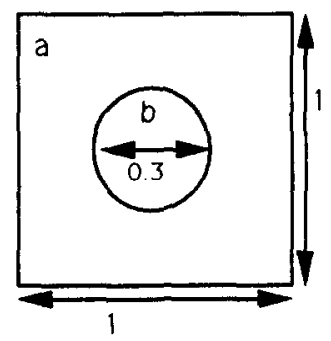

Fig. 8. Fiber-matrix composite. (a) Young's modulus: $72 \mathrm{GPa}$, Poisson's ratio: 0.32. (b) Young's modulus: $410 \mathrm{GPa}$, Poisson's ratio: 0.19 .
Table 3. Macroscopic coefficients (MPa) (fiber-matrix composite, plane stress hypothesis, $M=3, j=5$ )

$C^{\text {hum }}=\left[\begin{array}{ccc}116000 & 32400 & -1.94 \\ 32400 & 116000 & -1.94 \\ -1.94 & -1.94 & 38100\end{array}\right]$

Table 3 shows the numerical results and gives us a good accuracy relative to the finite element solution (see Table 4).

The fluctuations of the residuum are given in Fig. 9. The optimal value of the viscosity coefficient is equal to 0,01 .

An important remark is that the computation times are comparable for the two methods. The waveletGalerkin is more efficient because it is not necessary to generate a mesh. Without meshing, the finite element method ( $\mathrm{P} 1$ element) is more efficient, but, because of the choice of the order of the wavelet $(M=3)$, it could be necessary to compare with $\mathrm{P} 2$ elements. In this case, the number of computations for each element is four times larger.

\section{CONCLUSION}

Wavelet-Galerkin methods have been introduced for solving periodic homogenization problems. The numerical model presented in this paper turns out to be a good alternative to the finite element method.

An improvement to give to this work concerns the use of adaptivity properties. Then, it is necessary to introduce the wavelet function and not only the scale function. The computations are done too in the orthogonal complementary of the basis. The implementation is obviously more complicated.

Table 4. Macroscopic coefficients (MPa) (fiber-matrix composite, plane stress hypothesis, finite element method)

$C^{\text {hor }}=\left[\begin{array}{lcc}114000 & 32300 & -3.66 \\ 32300 & 114000 & -4.1 \\ 3.66 & -4.1 & 37400\end{array}\right]$

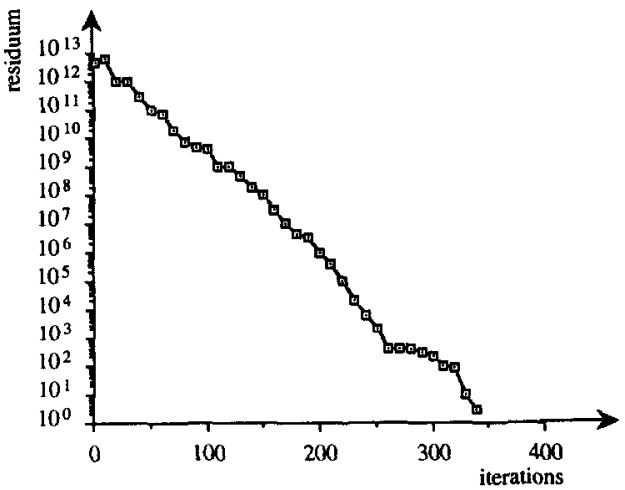

Fig. 9. Evolution of the residuum along the iterative process (fiber-matrix composite $M=3, j=5, \epsilon=0.01$, plane strain hypothesis) 


\section{REFERENCES}

1. Z. Hashin, The elastic moduli of heterogeneous materials. ASME J. Appl. Mech. 29, 143-150 (1962).

2. Z. Hashin and S. Strickman, A variational approach to the theory of the elastic behaviour of multiphase materials. J. Mech. Phys. Sol. 11, 127-140 (1963)

3. R. Hill, Theory of mechanical properties of fiberstrengthened materials. $J$. Mech. Phys. Sol. 12, 199-212 (1964).

4. J. Willis, Elasticity theory of composites. In: Mechanics of Solids, pp. 353-386. Pergamon Press, Oxford (1982)

5. P. Suquet, Plasticité et homogénéisation. Thesis, Université Paris 6 (1982).

6. C. Licht, Frottement, viscoplasticité et homogénéisation. Thesis, Université Montpellier 2 (1987).

7. F. Lenc, Contribution à l'étude des matériaux composites et de leur endommagement. Thesis, Université Paris 6 (1984).

8. H. Moulinec and P. Suquet, A fast numerical method for computing the linear and nonlinear mechanical properties of composites. C.R. Acad. Sci. Paris 318, $1417-1423$ (1994)

9. A. Bensoussan, J. L. Lions and G. Papanicolaou, Asymptotic Analysis for Periodic Structures. NorthHolland, Amsterdam (1978).

10. E. Sanchez-Palencia, Non Homogeous Materials and Vibration Theory. Springer, Berlin (1980).

11. L. Tartar, Estimations de coefficients homogénéisés. In Lecture Notes in Mathematics, Vol. 704, pp. 364-373. Springer, Berlin (1973).

12. H. Attouch, Viscosity solutions of minimization problems. Preprint 1994/07, Laboratory of Convex Analysis, Université Montepellier 2 (1994).

13. I. Daubechies, Orthonormal bases of compactly supported wavelets. Commun. Pure appl. Math. 41, $909-996$ (1988).

14. G. Beylkin, R. Coifman and V. Rokhlin, Fast wavelet transforms and numerical algorithms I. CPAMS 44, 141-183 (1991).

15. G. Beylkin, On the representation of operators in bases of compactly supported wavelets. SIAM J. mumer. Anal. 6, 1716-1760 (1992).

16. S. Dumont and F. Lebon, Ondelettes et équations aux dérivées partielles. I Le cas unidimentionnel. Note Interne no. 93-1, Laboratoire de Mécanique et Génie Civil, Université Montpellier 2 (1993).

17. J. Liandrat, V. Perrier and P. Tchamitchian, Numerical resolution of non linear partial differential equations using the wavelet approach. In: Wavelets and Their Applications (Edited by Ruskai et al.) pp. 227-238. Jones and Barlet, New York (1992).

18. R. Peyroux, C. Licht. Homogenization in thermoelasticity: application to composite materials. J. Phys. IV, Colloque C7, suppl. au $J$. Phys. III, 3, 1623-1626 (1993).

19. S. Dumont and F. Lebon, Representation of plane elastostatics operators in Daubechies wavelets (submitted)

20. H. Dumontet, Homogénéisation par développement en séries de Fourier. C.R. Acad. Sci. Paris 296, 1625-1628 (1983)

21. A. Cohen, Ondelettes et traitement numérique du signal, RMA 25, Masson, Paris (1992).

\section{APPENDIX: COMPUTATION OF $r_{0 O K}^{1}$}

To perform the coefficients $r_{00 k}^{1}$ it is necessary to determine an eigenvector of the matrix $\mathbb{D}$ with the eigenvalue equal to two.

Tables Al ( $M=3$ and $M=1)$ and A2 $(M=5$ and $\mathbb{M}=1)$ give the values of the vector $r_{004}^{\prime}$

Table Al. $r_{00 k}^{\mathrm{t}}$ for $(M=3$ and $\mathrm{M}=1)$

\begin{tabular}{cc}
\hline$k$ & $r$ \\
\hline 0 & 1.2863350694223 \\
1 & -1.6721720304760 \\
2 & 0.48110450704858 \\
3 & -0.091033200382807 \\
4 & -0.0042343456173233 \\
\hline
\end{tabular}

Table A2. $r_{00 k}^{1}$ for $(M=5$ and $M=1)$

\begin{tabular}{cc}
\hline$k$ & $r$ \\
\hline 0 & 0.69613605509369 \\
1 & -0.24707845028828 \\
2 & -0.63131161012272 \\
3 & 0.21948582822000 \\
4 & -0.035714247754115 \\
5 & -0.0032443713923207 \\
6 & 0.0017643657134024 \\
7 & -0.000037395342553624 \\
8 & -0.00000017407613287183 \\
\hline
\end{tabular}

